\title{
Earwax of patients with hidradenitis suppurativa: A retrospective study
}

\author{
Yuka Shibuya, Daichi Morioka, Misako Nomura, Zhuo Zhang, Hiroki Utsunomiya \\ Department of Plastic Surgery, Showa University, Tokyo, Japan
}

Background In Western nations, hidradenitis suppurativa (HS) typically affects the apocrine gland-bearing skin of people of African origin, women, smokers, and individuals with obesity. The clinical characteristics of HS in Korea and Japan, however, are reportedly different from those in the West. We therefore hypothesized that wet earwax is associated with HS because most East Asian people are genetically predisposed to produce dry earwax.

Methods The medical charts of 53 Japanese patients with HS were reviewed retrospectively. Results Unlike the results of surveys conducted in Western nations, most of our patients were men (72\%), whose buttocks were the most commonly affected site. Apocrine glandbearing areas, such as the axilla, were affected less often. The proportion of HS patients with wet earwax was $51 \%$, which was substantially higher than that found in the general Japanese population. Moreover, when patients with gluteal HS were excluded, the proportion of patients with wet earwax became even higher (68\%).

Conclusions Although the etiology of HS is unknown, our survey indicated that HS in apocrine gland-bearing skin, such as the axillary and anogenital areas, may be associated with wet earwax. As this study was conducted in a limited clinical setting, a nationwide, multicenter survey is warranted to clarify the clinical characteristics of HS in Japan.

Keywords Apocrine gland / Cerumen / Hidradenitis suppurativa / Medical chart review / Race factors
Correspondence: Daichi Morioka Department of Plastic Surgery, Showa University, 1-5-8 Hatanodai, Shinagawa-ku, Tokyo 142-8866, Japan

Tel: +81-3-3784-8548

Fax: +81-3-3784-9183

E-mail:dmorioka@gmail.com

Received: February 24, 2019 - Revised: September 2, $2019 \bullet$ Accepted: September 19, 2019

pISSN: 2234-6163 • elSSN: 2234-6171 • https://doi.org/10.5999/aps.2019.00290 • Arch Plast Surg 2019;46:566-571

\section{INTRODUCTION}

Hidradenitis suppurativa (HS) is a chronic, relapsing skin condition characterized by deep-seated nodules, boils, and abscesses that sometimes lead to sinus tract and fistula formation [1]. Although the etiology of HS is unclear, it is known to be a disorder of the sebaceous glands and terminal hair follicles of apocrine gland-bearing skin [2]. Due to the associated malodorous discharge, long-term pain, and scarring, the psychosocial functioning of patients with HS is often impaired. HS has a signifi- cant impact on patients' quality of life, professional activity, and self-esteem [3].

Several epidemiological studies of HS have been performed in Western nations, with the estimated prevalence of HS ranging from $0.05 \%$ to $4.0 \%$ [1]. HS predominantly affects obese women, and it typically occurs in the axillae, breasts, and anogenital areas of Caucasian and African individuals. It is less common in Hispanics and Asian people [4].

Contrary to these demographic data from Western nations, most Japanese patients with HS are men whose lesions are on 
the buttocks; relatively few of these patients present with lesions in apocrine-bearing areas, such as the axilla [5].

Wet earwax is a normal secretory product of the ceruminous apocrine glands, whereas dry earwax forms due to a genetically reduced number of apocrine glands [6,7]. A single-nucleotide polymorphism, $538 \mathrm{G}>\mathrm{A}$ in the $A B C C 11$ gene, determines the type of earwax that an individual produce [7]. G/A heterozygotes and G/G homozygotes produce wet earwax, whereas A/ A homozygotes produce dry earwax. Wet earwax has complete autosomal dominance over the dry type. Wet earwax is found in almost $100 \%$ of European and African populations, as well as in most (50\%-70\%) Southeast Asian, Oceanian, Central Asian, and Native American individuals. In contrast, dry earwax is highly specific to East Asian populations, with a frequency of $80 \%-95 \%[7,8]$. Only $12 \%$ of Japanese individuals produce wet earwax [6].

We therefore hypothesized that the clinical characteristics of Japanese patients with HS are different from those of patients in the West, and we placed a special emphasis on the type of earwax most often seen associated with this disease. We evaluated earwax type in a cohort of Japanese HS patients. In addition, we compared the clinical characteristics of patients with wet versus dry earwax.

\section{METHODS}

This study, which was conducted with the approval of the Institutional Review Board (IRB No. 2487) of Showa University, conforms to the standards established in the Declaration of Helsinki. Written consent for use of their photographs was obtained from all patients. Between May 2010 and June 2018, we retrospectively reviewed the medical charts of Japanese patients who met the criteria for an HS diagnosis according to standard guidelines (Table 1). Although most of the patients had been diagnosed with pyoderma chronica or acne conglobata, all also met the diagnostic criteria for HS. The patients' demographic information and clinical characteristics—sex, age of onset, family history of HS, affected area(s), and earwax type - were inves- tigated.

We particularly focused our statistical analyses on differences in the clinical characteristics of those with wet versus dry earwax. Variables were compared using the chi-square test. A value of $\mathrm{P}<0.05$ was considered to indicate statistical significance. All statistical analyses were performed using Microsoft Excel 2013 (Microsoft Corp., Redmond, WA, USA).

\section{RESULTS}

In total, we reviewed the medical charts of 53 patients. Table 2 shows their demographic data and clinical characteristics. Of the 53 patients, 38 were male $(71.7 \%)$ and 15 were female (28.3\%). The mean age at onset of HS was 32.4 years, and the mean duration of the disease was 7.6 years. Patients with obesity

\section{Table 2. Patients' characteristics $(n=53)$}

\begin{tabular}{|lc|}
\hline Characteristic & No. (\%) \\
\hline Sex & \\
Male & $38(71.7)$ \\
Female & $15(28.3)$ \\
Mean age at disease onset (yr) & 32.4 \\
Mean disease duration (yr) & 7.6 \\
Body mass index (kg/m $\left.{ }^{2}\right)$ & \\
$<30$ & $46(86.8)$ \\
$\geq 30$ & $7(13.2)$ \\
Smoking status & \\
Current or former smoker & $45(84.9)$ \\
$\quad$ Nonsmoker & $8(15.1)$ \\
Positive family history of hidradenitis suppurativa & $16(30.2)$ \\
Affected areas $\left.{ }^{a}\right)$ & \\
Gluteal & $22(41.5)$ \\
Anogenital/inguinal & $16(30.2)$ \\
Periauricular & $15(28.3)$ \\
Axillary & $9(17.0)$ \\
Mammary & $2(3.8)$ \\
Otherb) & $5(9.4)$ \\
Type of earwax & \\
Wet & $27(50.9)$ \\
Dry & $26(49.1)$ \\
\hline a) Some patients suffered from hidradenitis suppurativa in two or more bodily \\
areas; b) Other areas include the chin, nape of the neck, shoulder, back, and thigh. \\
\end{tabular}

Table 1. Diagnostic criteria for hidradenitis suppurativa [2]

Primary positive diagnostic criteria

History: painful or suppurating lesions that recurred more than twice in 6 months

Signs: involvement of the axilla, genitofemoral area, perineum, gluteal area, or (in women) the inframammary area; presence of nodules (inflamed or non-inflamed), sinus tracts (inflamed or non-inflamed), abscesses, or scarring (atrophic, mesh-like, red, hypertrophic, or linear)

Secondary positive diagnostic criteria

History: family history of hidradenitis suppurativa

Microbiology: a negative swab or the presence of normal skin microbiota may be indicative of hidradenitis suppurativa 
(characterized by a body mass index $\geq 30 \mathrm{~kg} / \mathrm{m}^{2}$ ) comprised $13.2 \%$ of the 53 patients. Overall, $84.9 \%$ were current or former smokers. A family history of HS was found in $30.2 \%$ of the second-degree relatives of the patients. Some patients had HS that affected two or more areas of the body. The gluteal area was most often affected (Fig. 1), followed by the anogenital/inguinal and periauricular areas. Only nine patients had lesions in the axillae (Fig. 2). Other uncommon areas of HS occurrence included the chin, nape of the neck, shoulder, back, and thigh. Wet earwax was observed in 27 of the 53 patients, whereas dry earwax was observed in 26 . There were no significant differences in these variables between the sexes.

When the clinical characteristics of patients with wet and dry earwax were compared, no significant differences were found regarding sex, age at onset of HS, disease duration, smoking sta- tus, or body mass index. As shown in Table 3, however, 14 of the 27 patients with wet earwax had a positive family history of HS, whereas only two of the 26 patients with dry earwax had such a history $(\mathrm{P}<0.001)$.

We divided the patients into two groups: a "gluteal" group, consisting of patients who had lesions on their buttocks only $(\mathrm{n}=15)$, and an "other-areas" group, consisting of all other patients $(\mathrm{n}=38)$. Among the 27 patients with wet earwax, two had HS only on their buttocks. In contrast, among the 26 patients with dry earwax, 13 were categorized into the gluteal group, while 13 had HS on other areas $(\mathrm{P}<0.01)$. Thus, the gluteal group predominantly included those with dry earwax (13 of 15 patients), whereas the other-areas group predominantly included those with wet earwax (25 of 37 patients).

\section{Fig. 1. Typical cases of hidradenitis suppurativa}

Both patients were current smokers. (A) A 51-year-old man presented with Hurley stage III hidradenitis suppurativa (HS) on the buttocks only. The patient had dry earwax and no family history of HS. (B) A 54-year-old man presented with Hurley stage III HS in the perianal area that extended into the gluteal area, as well as stage II HS in the periauricular area. The patient had wet earwax and had two daughters with HS.
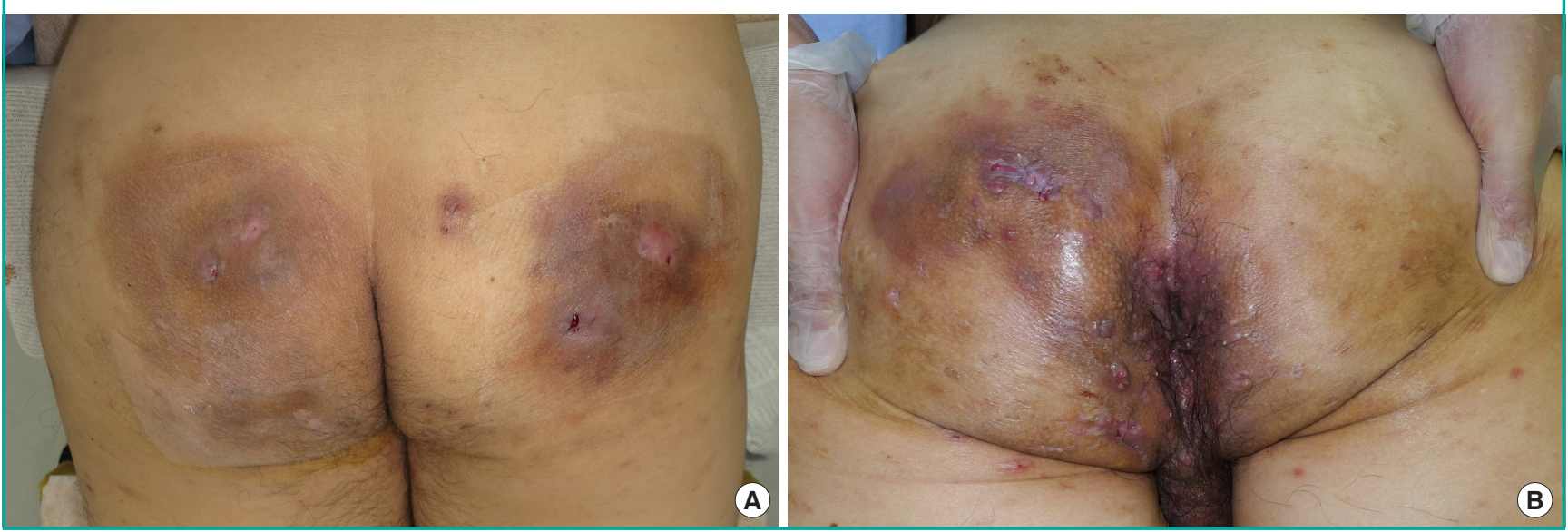

Fig. 2. Other cases photographs of hidradenitis suppurativa

(A) A 32-year-old man presented with Hurley stage II hidradenitis suppurativa on the periauricular region. (B) A 25-year-old man presented with Hurley stage II hidradenitis suppurativa on the bilateral axillae. Both patients were current smokers and had wet earwax.
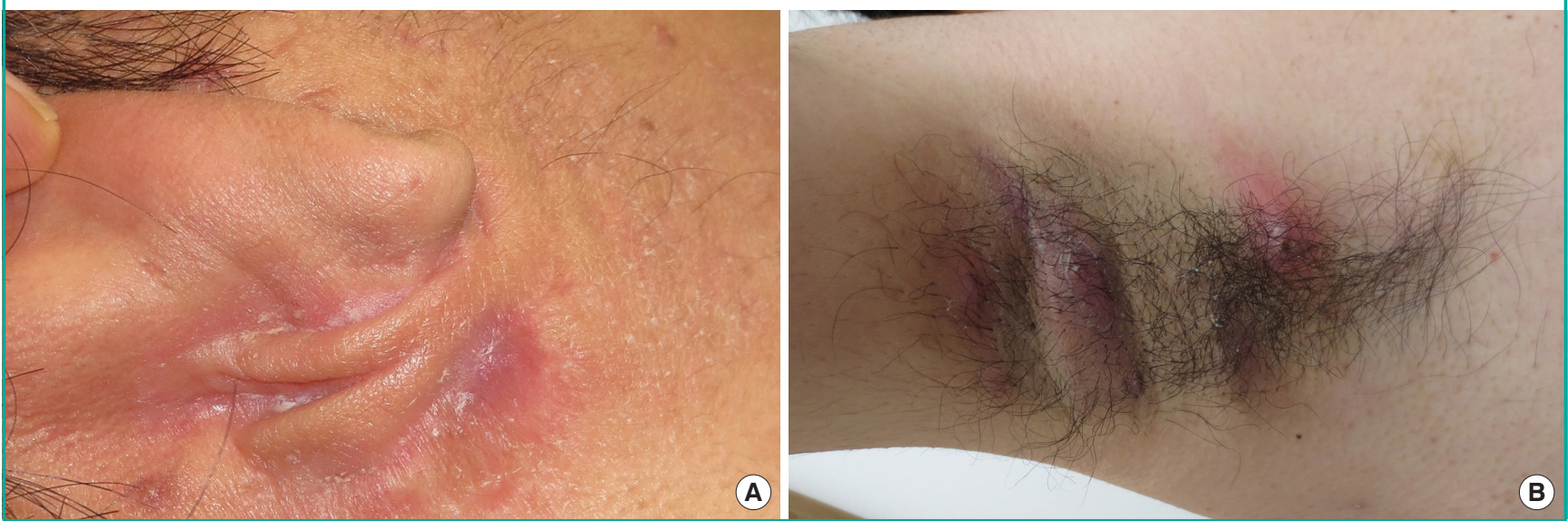
Table 3. Patients' characteristics according to the presence of wet or dry earwax

\begin{tabular}{|c|c|c|c|}
\hline Characteristic & $\begin{array}{c}\text { Wet } \\
\text { earwax } \\
(n=27)\end{array}$ & $\begin{array}{c}\text { Dry } \\
\text { earwax } \\
(n=26)\end{array}$ & P-value ${ }^{a)}$ \\
\hline Family history of hidradenitis suppurativa & & & $<0.001$ \\
\hline Yes & 14 & 2 & \\
\hline No/unknown & 13 & 24 & \\
\hline Affected area & & & $<0.01$ \\
\hline Buttocks only & 2 & 13 & \\
\hline Other sites ${ }^{\text {b) }}$ & 25 & 13 & \\
\hline
\end{tabular}

Values are presented as number.

a'These values were obtained using the chi-square test; b Details of the other sites are described in the Methods section. Six of these patients experienced hidradenitis suppurativa on their buttocks as well.

\section{DISCUSSION}

HS has traditionally been viewed as a chronic, relapsing inflammatory disorder of the sweat glands. Although most HS-affected areas are apocrine gland-rich, these glands are not the primary target of the pathological process of the disease [9]. The involvement of apocrine glands in HS is still under debate, as not only apocrine-rich areas but also intertriginous areas (e.g., the inframammary fold) are commonly affected. The pathogenesis of HS is currently thought to be multifactorial and likely heterogeneous [9]. Previously reported risk factors include being of African or Caucasian origin, being female, smoking, and obesity [2]. An autosomal dominant inheritance pattern is observed in one-third of HS patients [1].

The wet variety of earwax has complete autosomal dominance over the dry type. In this regard, Japan is unique in that only $12 \%$ of individuals have wet earwax [6]. Hence, it is noteworthy that half of the Japanese patients with HS in the present study were found to have wet earwax. Excluding the gluteal group, 25 of 37 patients had wet earwax (resulting from the $\mathrm{G}$ allele of the $A B C C 11$ gene), which may be another risk factor for HS in this group. It could be interesting to investigate the earwax type resulting from the $A B C C 11$ genotype in Asian and Hispanic patients with $\mathrm{HS}$.

Atypical histopathological findings are common in HS. These include intrafollicular hyperkeratosis, which causes plugging and rupture of the hair follicles, followed by perifollicular lymphocytic infiltration, abscess formation, sinus tracts with stratified squamous epithelium, and fibrosis $[2,10]$. In this study, we found no histological differences between the lesions of patients with wet earwax and those with dry earwax because all patients had completely disrupted, non-functioning sweat glands.

Anatomically, the distribution of apocrine glands is limited to the ear canals, axillae, breasts, and perianal area. Apocrine glands are not found in the skin of the buttocks. In a few studies conducted in the West, the clinical features of patients with gluteal HS were found to be different from those of other subgroups [11]. Canoui-Poitrine et al. [11] divided HS into three subgroups—axillary-mammary, follicular, and gluteal—and reported that gluteal $\mathrm{HS}$ was predominant in males and was associated with smoking, but not with obesity $[1,11]$. Similarly, in the present study, patients characterized by lesions only in the apocrine gland-poor buttock area were predominantly men and smokers to a statistically significant degree. Only one patient had a family history of HS. In addition, the proportion of patients with wet earwax ( 2 of 16 patients) was approximately equivalent to that in the general Japanese population.

In Korea, $\leq 5 \%$ of individuals are known to have wet earwax $[7,8]$. Interestingly, similarly to Japanese HS patients, Korean HS patients are predominantly male, and the most commonly affected area in these patients is also the buttocks [12]. The recently reported prevalence of HS in Korea was low $(0.06 \%)$ [13]. Although the prevalence of HS in Japan has not been investigated, Kurokawa et al. [5] surmised that it was lower than that in Western nations.

In Western nations, the sites most commonly affected by HS are the inguinal and axillary areas, with the gluteal area being only the third most common site [1]. We suggest that both Japanese and Korean patients with HS are most commonly affected on their buttocks. Thus, patients in Japan and Korea are less likely to be affected in their apocrine gland-bearing skin areas, such as the axillae, than are either Africans or Caucasians. As shown in Table 2, when patients who exhibited HS only on their buttocks were excluded, the frequency of a family history of HS was significantly higher, and the proportion of patients with wet earwax ( 25 of 37 patients) was extremely high. As previously noted, the pathogenesis of HS is multifactorial [9]. Gluteal HS may be more closely associated with environmental factors (e.g., cigarette smoking), whereas lesions in apocrine glandbearing skin may be associated more strongly with genetic factors. In fact, some physicians and surgeons prefer to diagnose gluteal symptoms as pyoderma chronica glutealis rather than gluteal HS [5].

Previous studies have led to the proposition of several HS subtypes $[11,14]$. As clinical characteristics and pathogenesis differ by subtype, the optimal treatment of the subtypes may also be different [14]. Canoui-Poitrine et al. [11] reported that cases of gluteal HS were less severe than those of other subtypes. General advice to stage I HS patients includes lifestyle modification, weight control, and smoking cessation. For such mild lesions, Lee et al. [13] recommended topical or oral administration of 
antibiotics prior to incision and drainage of the lesion. Administration of some hormones, such as antiandrogens and corticosteroids, has also been recommended [2]. For moderate to severe lesions, radical excision of affected areas and the surrounding tissues is the most common treatment $[2,10]$. The defects are primarily closed, then treated using negative-pressure wound healing therapy. Alternatively, they are reconstructed with skin grafting or local flaps. Deroofing of sinus lesions and carbon dioxide laser treatments are also used [10]. Recently, a human monoclonal antibody to tumor necrosis factor alpha proved to be an efficacious treatment for moderate to severe HS in Western patients [2]. For the reasons that we have indicated, physicians examining patients suspected of having HS and investigators in charge of clinical trials should take the type of earwax of the patient into account.

As we hypothesized, the present study indicated that wet earwax was associated with HS. However, this study consisted of subjects who were referred for plastic surgery to a limited number of treatment centers in central Tokyo. Based on our results, a nationwide, multicenter survey is warranted to clarify the clinical features of Japanese patients with HS. We believe that East Asian nations, including Japan and Korea, could provide suitable populations for studies to determine the association of wet earwax and/or the $A B C C 11$ gene with HS.

\section{NOTES}

\section{Conflict of interest}

No potential conflict of interest relevant to this article was reported.

\section{Ethical approval}

The study was approved by the Institutional Review Board of Showa University (IRB No. 2487) and performed in accordance with the principles of the Declaration of Helsinki. Written informed consents were obtained.

\section{Patient consent}

The patients provided written informed consent for the publication and the use of their images.

\section{Author contribution}

Conceptualization: Shibuya Y, Morioka D. Data collection: all authors. Statistical analysis: Shibuya Y, Utsunomiya H, Zhang Z. Artwork: Shibuya Y, Nomura M, Utsunomiya H. Writing: Shibuya Y, Morioka D. Approval of final manuscript: all authors.

\section{ORCID}

Yuka Shibuya https://orcid.org/0000-0003-4114-4724

Daichi Morioka https://orcid.org/0000-0002-6210-9808

Misako Nomura https://orcid.org/0000-0002-1816-9926

Zhuo Zhang https://orcid.org/0000-0002-1914-1722

Hiroki Utsunomiya https://orcid.org/0000-0003-2428-6931

\section{REFERENCES}

1. Parulkar I, Haleem H, Paek SY. Epidemiologic and clinical features of hidradenitis suppurativa. Semin Cutan Med Surg 2017;36:42-6.

2. Zouboulis CC, Desai N, Emtestam L, et al. European S1 guideline for the treatment of hidradenitis suppurativa/acne inversa. J Eur Acad Dermatol Venereol 2015;29:619-44.

3. Ingram JR, Jenkins-Jones S, Knipe DW, et al. Populationbased Clinical Practice Research Datalink study using algorithm modelling to identify the true burden of hidradenitis suppurativa. Br J Dermatol 2018;178:917-24.

4. Udechukwu NS, Fleischer AB Jr. Higher risk of care for hidradenitis suppurativa in African American and non-Hispanic patients in the United States. J Natl Med Assoc 2017;109:448.

5. Kurokawa I, Hayashi N; Japan Acne Research Society. Questionnaire surveillance of hidradenitis suppurativa in Japan. J Dermatol 2015;42:747-9.

6. Super Science High School Consortium. Japanese map of the earwax gene frequency: a nationwide collaborative study by Super Science High School Consortium. J Hum Genet 2009; 54:499-503.

7. Nakano M, Miwa N, Hirano A, et al. A strong association of axillary osmidrosis with the wet earwax type determined by genotyping of the ABCC11 gene. BMC Genet 2009; 10:42.

8. Yoshiura K, Kinoshita A, Ishida T, et al. A SNP in the $\mathrm{ABCC} 11$ gene is the determinant of human earwax type. Nat Genet 2006;38:324-30.

9. Kurzen H, Kurokawa I, Jemec GB, et al. What causes hidradenitis suppurativa? Exp Dermatol 2008;17:455-6.

10. Danby FW, Margesson LJ. Hidradenitis suppurativa. Dermatol Clin 2010;28:779-93.

11. Canoui-Poitrine F, Le Thuaut A, Revuz JE, et al. Identification of three hidradenitis suppurativa phenotypes: latent class analysis of a cross-sectional study. J Invest Dermatol 2013; 133:1506-11.

12. You HR, Yun SJ, Lee SC, et al. Clinical characteristics and epidemiology of hidradenitis suppurativa in Korea: a singlecenter study. Korean J Dermatol 2016;54:723-7.

13. Lee JH, Kwon HS, Jung HM, et al. Prevalence and comor- 
bidities associated with hidradenitis suppurativa in Korea: a nationwide population-based study. J Eur Acad Dermatol Venereol 2018;32:1784-90.
14. van der Zee HH, Jemec GB. New insights into the diagnosis of hidradenitis suppurativa: clinical presentations and phenotypes. J Am Acad Dermatol 2015;73(5 Suppl 1):S23-6. 